\title{
Guia ilustrado para mediar educação em saúde com pessoas após o acidente vascular cerebral: construção e validação de conteúdo
}

\author{
Illustrated guide to mediate health education with people after stroke: construction and \\ content validation
}

Guía ilustrada para mediar en la educación sanitaria con personas después del accidente cerebrovascular: construcción y validación de contenido

Renata de Oliveira Galvão ${ }^{1}$ Elizabeth Teixeira ${ }^{1 *}$, Camila Rodrigues Barbosa Nemer².

\section{RESUMO}

Objetivo: validar o conteúdo de um guia ilustrado para mediar educação em saúde com pessoas após o acidente vascular cerebral. Métodos: pesquisa metodológica, constituída em revisão da literatura; construção do guia ilustrado; e, validação de conteúdo com 19 juízes-especialistas da área da saúde e de outras áreas. Foram aplicados dois questionários distintos, os dados em estatística descritiva passaram para análise quantitativa no Índice de Validade de Conteúdo e no Escore SAM. Resultados: O Índice de Validade de Conteúdo encontrado foi de 0,79. O Escore SAM foi de 26 pontos. As sugestões obtidas dos juízesespecialistas indicaram ser necessário mais informações sobre afecções crônicas e autocuidado, bem como a inclusão de informações sobre disfagia e marcha, em função da qualidade de vida após evento vascular. Conclusão: posterior a validação, o guia ilustrado evidenciou-se um dispositivo adequado para mediar ações de educação em saúde com pessoas acometidas por alterações neurológicas devido ao acidente vascular cerebral.

Palavras-chave: Tecnologia educacional, Educação em saúde, Infarto cerebral, Estudos de validação.

\begin{abstract}
Objective: to validate the content of a illustrated guide to mediate health education with people after stroke. Methods: methodological research, consisting of a literature review; construction of the illustrated guide; and, content validation with 19 expert judges from the health and other areas. Two different questionnaires were applied, the data in descriptive statistics passed for quantitative analysis in the Content Validity Index and the SAM Score. Results: The Content Validity Index found was 0.79 . The SAM score was 26 points. The suggestions obtained from the expert judges indicated that more information on chronic conditions and selfcare is needed, as well as the inclusion of information on dysphagia and gait, depending on the quality of life after a vascular event. Conclusion: after validation, the illustrated guide proved to be an adequate device to mediate health education actions with people affected by neurological changes due to stroke.
\end{abstract}

Keywords: Educational technology, Health education, Cerebral infarction, Validation studies.

RESUMEN

Objetivo: validar el contenido de una guía ilustrada para mediar la educación sanitaria con personas después de un accidente cerebrovascular. Métodos: investigación metodológica, que consiste en una revisión de la literatura; construcción de la guía ilustrada; y validación de contenido con 19 jueces expertos de la salud y

1 Universidade do Estado do Amazonas (UEA), Manaus-AM. *E-mail: etfelipe@hotmail.com

2 Universidade Federal do Amapá (UNIFAP), Macapá - AP.

Financiamento pela Universidade do Estado do Amazonas (UEA) / Fundação de Amparo à Pesquisa do Estado do Amazonas (FAPEAM), Programa de Iniciação Científica e Tecnológica da Universidade do Estado do Amazonas (PAIC-AM), processo no 14961. 
otras áreas. Se aplicaron dos cuestionarios diferentes, los datos en estadística descriptiva pasaron para el análisis cuantitativo en el Índice de Validez de Contenido y el Puntaje SAM. Resultados: El índice de validez de contenido encontrado fue de 0,79. El puntaje SAM fue de 26 puntos. Las sugerencias obtenidas de los jueces expertos indicaron que se necesita más información sobre afecciones crónicas y autocuidado, así como la inclusión de información sobre disfagia y marcha, según la calidad de vida después de un evento vascular. Conclusión: después de la validación, la guía ilustrada demostró ser un dispositivo adecuado para mediar las acciones de educación sanitaria con personas afectadas por cambios neurológicos debido a un accidente cerebrovascular.

Palabras clave: Tecnología educativa, educación sanitaria, infarto cerebral, estudios de validación.

\section{INTRODUÇÃO}

As doenças crônicas não transmissíveis (DCNT) configuram uma questão de saúde preocupante em escala mundial, devido as diversas perdas e o elevado número de mortes, cerca de $70 \%$ no total (MALTA DC, et al., 2017). Por essa razão o Plano de Enfrentamento das DCNT 2011-2022 foi delineado pelo Ministério da Saúde do Brasil com propósito de conter os agravos a longo prazo no país. A estratégia efetiva do plano é estruturada em eixos: principalmente a manutenção do cuidado integral, a viabilização da promoção da saúde e a realização da constante vigilância com frequente avaliação, regular monitoramento e acesso permanente da informação. (BRASIL, 2011). Nesse contexto, o eixo do cuidado integral tem como proposta fortalecer o campo da educação em saúde voltada para as quatro DCNT de maior impacto, respectivamente, doenças cardiovasculares, diabetes, neoplasias e doenças respiratórias crônicas (BRASIL, 2011).

A educação em saúde está vinculada ao processo saúde-doença em que a transmissão do conhecimento tem sido pautada por concepções alternantes entre o eixo vertical-individual e o horizontal-coletivo (TEIXEIRA E e OLIVEIRA DC, 2014; TEIXEIRA, E, 2020). Abordagens verticalizadas enfatizam o conteúdo fornecido pelo indivíduo ao qual agrega não só recomendações clínicas e orientações práticas, mas também hábitos de vida, comportamentos e opiniões. Além disso, absorvem experiências com vieses significativos, e assim responsabiliza o sujeito pela adaptação às condutas, uma vez que nessas circunstâncias a educação em saúde se fundamentada na informação (TEIXEIRA E e OLIVEIRA DC, 2014; TEIXEIRA, E, 2020).

Em contrapartida, abordagens horizontalizadas reforçam a construção das informações que incentivam a dialética sobre ações questionadoras, legitimam as situações emancipatórias, sustentam intervenções coletivas todas como meios transformadores da realidade. Elas relacionam expectativas e necessidades públicas, princípios e entendimentos culturais, sem descaracterizar o sujeito e seus anseios. Nessa conjuntura, a educação em saúde se embasa no conteúdo e na produção em grupo (TEIXEIRA E e OLIVEIRA DC, 2014; TEIXEIRA, E, 2020).

É essencial reforçar que abordagens horizontalizadas podem ser mediadas por tecnologias educacionais produzidas a partir de evidências. Tais tecnologias podem apoiar as práticas educativas em saúde realizadas pelos profissionais de saúde. Apesar disso, por um lado as tecnologias educacionais em saúde são produzidas, por outro lado a maioria não é validada nem avaliada. Ressalta-se ainda que diversas tecnologias impressas raramente são submetidas a um processo de validação. Isso se correlaciona ao fato de muitos profissionais não saberem realizar o procedimento de validação e disponibilizam diretamente para a população materiais não testados e não validados (MOREIRA APA, et al., 2014).

O infarto cerebral, foco desse estudo em forma de estratégia educacional, integra o plano contra DCNT compreendido no subitem c "linha do cuidado acidente vascular encefálico (AVE) na rede de atenção às urgências" no tópico de ação $V$ "Saúde toda hora" no terceiro eixo "cuidado integral" (BRASIL, 2011). O acidente encefálico apresenta início abrupto de sintomas e sinais, desencadeia a perda de função cerebral focal ou global por um período maior que 24 horas (BRONSON RJ e CIFU AD, 2019). Desse modo, trata-se de uma patologia na condição clínica de tempo crítico como um evento agudo, porém permeia as principais causas de inabilidades a longo prazo com manifestações e evoluções crônicas (STEIGER N e CIFU AS, 2016). 
Epidemiologicamente, o AVC é não só a primeira causa de morte no Brasil e a segunda no mundo, mas também a principal causa de incapacidade funcional na população economicamente ativa, desencadeando grande impacto socioeconômico (OPAS/OMS, 2019; BRASIL, 2017; ALVES N e PAZ FA, 2018). A estimativa mundial de sobreviventes se aproxima de 50 milhões, desses em média 49,5\% (25 a 75\%) podem evoluir com alterações emocionais, cognitivas ou físicas e assim irão demandar auxílio para efetivar suas atividades diárias (SCHMIDT MH, et al., 2019).

A análise de dados de um estudo realizado no Sul do Brasil apontou que $76 \%$ dos sobreviventes apresentaram alterações resultantes do evento cerebral como hemiplegia esquerda $(38,4 \%)$ e direita $(26,4 \%)$, quadriplegia $(3,2 \%)$, ataxia $(3,2 \%)$, paralisia facial direita $(3,2 \%)$, associada também a alterações oftálmicas e afasias (SÁ BP, et al., 2014).

Esse quadro clínico tanto requer o fortalecimento do autocuidado por parte da pessoa vivendo com o AVC, quanto da rede de apoio composta por cuidadores e familiares focada na autonomia, além da integração participativa de condutas no aprimoramento do melhor viver desse núcleo familiar. Práticas educativas em saúde nesse sentido devem ser realizadas, e assim, é preciso que existam tecnologias educacionais validadas para subsidiar o agir dos clientes, cuidadores, familiares e profissionais de saúde, no âmbito da reabilitação desses indivíduos que manifestam dificuldades ou perdas de funções. A motivação para a construção e validação de uma tecnologia educacional (TE) sobre o viver bem após um AVC foi pautada nesses aspectos voltados para os sobreviventes, cuidadores e familiares.

O objetivo do estudo foi desenvolver e validar o conteúdo de um guia ilustrado para mediar educação em saúde para pessoas com repercussões clínicas após o acidente vascular cerebral e para seus familiares.

\section{MÉTODOS}

Trata-se de uma pesquisa metodológica ao qual utiliza conhecimentos existentes com o propósito de gerar uma nova intervenção, melhorar significativamente uma existente e/ou melhorar um instrumento (TEIXEIRA E e NASCIMENTO MHM, 2019). Foi desenvolvida em três etapas: revisão da literatura, construção do guia ilustrado e validação de conteúdo. O estudo foi realizado no período de agosto de 2018 a julho de 2019 tendo como instituição âncora uma universidade localizada em um município do Amazonas.

\section{Fase 1: Revisão de literatura}

A revisão foi realizada na Biblioteca Virtual de Saúde. O descritor de busca segundo o $\mathrm{MeSH}$ foi sequelas de AVC com o filtro reabilitação do acidente vascular cerebral, selecionados pelos focos qualidade de vida e autocuidado. Foram adotados os seguintes critérios de inclusão: 2009-2019, texto completo; ensaio clínico controlado, revisões sistemáticas e guia de prática clínica; responder à questão da revisão pela técnica população, intervenção, contexto-PICo. Os critérios de exclusão foram: não relacionado ao tema, sem aplicabilidade prática direta (técnicas intermediadas por computadores e/ou programas), pesquisa em animais ou escalas de classificação ou avaliação.

\section{Fase 2: Construção do Guia llustrado}

Com base nos temas geradores obtidos com a revisão de literatura, foram elencados os conteúdos preliminares, a organização e o layout do guia com ilustrações. Após, foi desenvolvida a primeira versão por meio do processo de edição e diagramação, de acordo com critérios relacionados ao conteúdo (pertinentes ao autocuidado apoiado) e estrutura-organização (formato digital e impresso), com atenção aos aspectos de adequação de linguagem ao público, sensibilidade cultural e design gráfico.

\section{Fase 3: Validação de Conteúdo}

Participaram como juízes-especialistas (JE) profissionais de saúde e de outras áreas (comunicação, design gráfico e instrucional). Todos os contatos foram realizados por meio de ambiente virtual (contato eletrônico via e-mail). A amostra foi do tipo não probabilística intencional, utilizando-se do recurso snowball. 
Os critérios de inclusão foram: alcançar dois escores entre os itens: ser da saúde com expertise no âmbito de atendimento a pacientes com AVC (consulta à Plataforma Lattes); 3 anos de experiência na área; publicação científica sobre o AVC; e/ou participação na sociedade da área. Para os JE outras áreas: expertise no âmbito das TE; 3 anos de atuação em áreas relacionada a confecção de TE; publicação científica sobre o TE; e/ou, 3 anos de atuação na área desenvolvida. O critério de exclusão foi não responder contato eletrônico por 20 dias no período da coleta de dados.

O contato com os juízes ocorreu da seguinte maneira: primeiro e-mail com convite para participar da pesquisa; segundo e-mail com documento sobre o método da pesquisa, o Termo de Consentimento Livre e Esclarecido (TCLE) e o link disponível para assinar eletronicamente o TCLE pela plataforma "Google Formulários"; terceiro e-mail com a tecnologia educacional (o guia ilustrado) em formato PDF e o instrumento de avaliação via link pela plataforma "Google Formulários". Um instrumento específico para cada tipo de JE. (saúde e outras áreas).

O instrumento para os juízes-especialistas da área da saúde foi validado e tem como foco a análise de constructo ou de conteúdo da TE (NASCIMENTO MHM e TEIXEIRA E, 2018). Está organizado em três partes: identificação, orientação de preenchimento e escala tipo Likert. É composto por um conjunto de frases (itens) e se pede ao juiz-especialista para manifestar o grau de concordância de 1 a 4 . $O$ instrumento possui a seguinte estrutura de escala: totalmente adequado (1), adequado (2), parcialmente adequado (3) e inadequado (4).

O instrumento para os juízes-especialistas de outras áreas foi adaptado para se obter análise de aparência e interface comunicacional. (NASCIMENTO MHM e TEIXEIRA E, 2018). O instrumento com escore SelfAssessment Manikin (SAM) está organizado da seguinte maneira: adequado (2), parcialmente adequado (1) e inadequado (0).

A análise dos dados dos JE da saúde foi realizada com base no Índice de Validade de Conteúdo (IVC) calculado por meio do somatório de concordâncias dos itens marcados como "1" e "2", dividido pelo total de respostas. A validação da TE é considerada positiva se alcançar um IVC igual ou superior a 0,7 (NASCIMENTO MHM e TEIXEIRA E, 2018). A análise dos dados oriundos dos JE de outras áreas é equacionada pelo Escore SAM. A TE é validada se obtiver um Escore SAM igual ou superior a 10 pontos.

O projeto foi submetido via Plataforma Brasil e foi aprovado pelo Comitê de Ética em Pesquisa (CAAE 88932418.3.0000.514).

\section{RESULTADOS}

Em relação à revisão da literatura, foram selecionados 21 artigos com a temática reabilitação e qualidade de vida pós-evento vascular cerebral que contemplavam propostas de fornecer autonomia e autocuidado ao sobrevivente e família. Da leitura exaustiva emergiram os seguintes temas geradores: insônia, depressão, ansiedade, linguagem, movimentos, negligência, cuidador.

Em relação ao processo de construção, o protótipo do guia ilustrado, "Guia AVC para você e familiares", foi desenvolvido em 9 tópicos e ao final ficou com 23 páginas. Foi organizado em duas partes: "I. O que é AVC e quais suas consequências"; "II. A vida após o AVC" com a proposta de explicar as alterações cerebrais e orientar na reabilitação.

Foi elaborado com linguagem simplificada contendo infográficos, textos curtos e tabelas. A primeira versão foi disponibilizada aos JE no formato digital e no impresso (papel couchê tamanho A3, dobrado em tamanho A4, estilo revista).

Para o processo de validação de conteúdo, foram selecionados 14 juízes especialistas na área da saúde (Medicina: especialistas em Neurologia, Urgência e Emergência e Psiquiatria; Enfermagem: especialistas em Neurologia, Terapia Intensiva, Intervencionismo e Saúde Coletiva; Fonoaudiologia; Fisioterapia; Terapia ocupacional; e, Psicologia.) e 5 profissionais de outras áreas (Pedagogia, Publicidade e Propaganda, Direção de Arte, Ciências Sociais e Comunicação e especialista técnica em TE). 
Após a devolução dos instrumentos preenchidos e análise das respostas, obteve-se com os juízes especialistas da área de saúde um IVC de 0,79, resultado da média aritmética segundo os blocos do instrumento: objetivos 0,78; estrutura e apresentação 0,77; e, relevância 0,82 (Tabelas 1, 2 e 3). Com o instrumento utilizado por juízes-especialistas de outras áreas, obteve-se um Escore SAM de 26 pontos (Tabela 4).

Tabela 1 - Avaliação de Juízes Especialistas de Área Saúde na tecnologia educacional "Guia AVC para você e familiares", segundo os objetivos em Escala Likert.

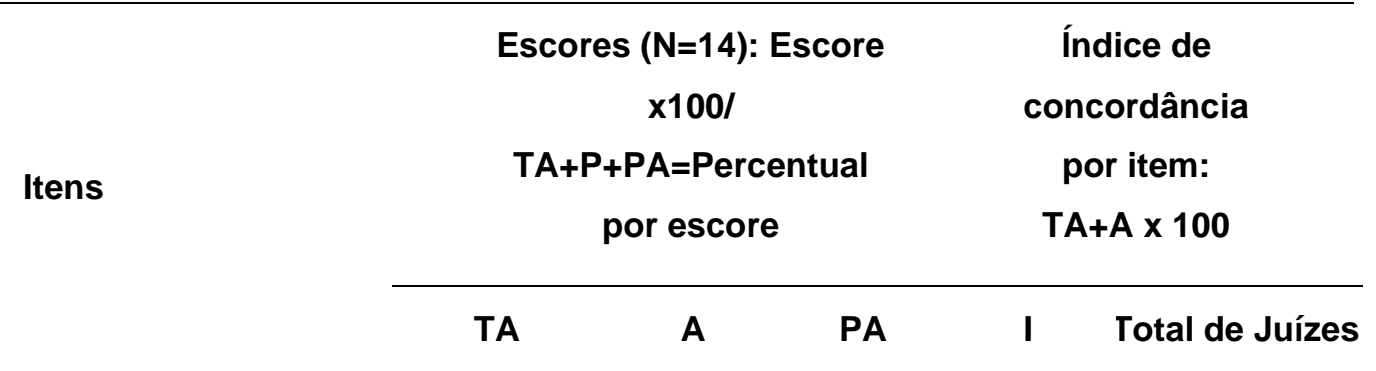

\section{Bloco 1. Objetivos}

1.1 As informações/conteúdos são ou estão coerentes com as necessidades 3 5 5 0,57 cotidianas do público-alvo da TE

1.2 As informações/conteúdos são importantes para a qualidade de 9 4 1 0 0,92 vida/trabalho do público-alvo da TE

1.3 A TE convida e/ou instiga as mudanças de comportamento, hábitos e 6 7 0 1 0,92 atitudes

1.4 A TE pode circular no meio científico da área

6 5

3

0

0,78

1.5 A TE atende aos objetivos que se propõe atingir com o público-alvo da TE

$\begin{array}{lllll}8 & 2 & 3 & 1 & 0,71\end{array}$

\begin{tabular}{lccccc}
\hline Escore por bloco & 32 & 23 & 12 & 3 & 55 \\
\hline Percentual por bloco & 45,71 & 32,86 & 17,14 & 4,28 & 78,57 \\
\hline IVC & & & 0,78 \\
\hline Total dos percentuais & 99,99 & & 78,57 \\
\hline
\end{tabular}

Legenda: Escores TA=Totalmente adequado; $\mathrm{A}=\mathrm{Adequado}$; $\mathrm{PA}=$ Parcialmente adequado; $\mathrm{I}=$ =Inadequado; IVC=Índice de Validação de conteúdo. Fonte: GALVAO RO, et al., 2020. 
Tabela 2 - Avaliação de Juízes Especialistas de Área Saúde na tecnologia educacional "Guia AVC para você e familiares", segundo estrutura e apresentação em Escala Likert.

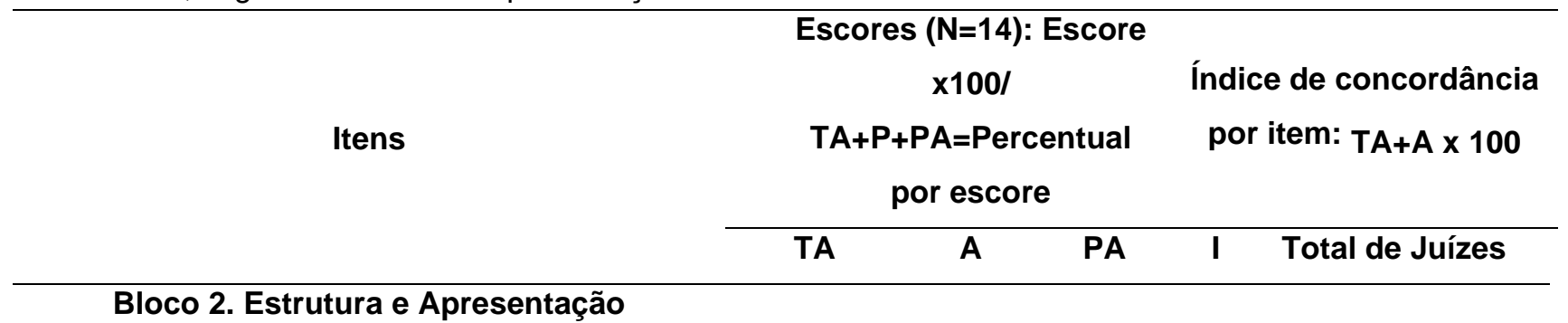

2.1 A TE é apropriada ser usada pelo público-

$$
\text { alvo }
$$

4

2.2 As mensagens estão apresentadas de maneira clara e objetiva

2.3 As informações apresentadas estão cientificamente corretas

2.4 O material está apropriado ao nível sociocultural do público alvo da TE

2.5 Há uma sequência lógica do conteúdo

$$
\text { proposto }
$$

2.6 As informações estão bem estruturas em concordância e ortografia

2.7 O estilo da redação corresponde ao nível de conhecimento do publico alvo

2.8 As informações do pré-texto, como capa, contracapa, sumário, agradecimentos e/ou apresentação são adequadas

2.9 O tamanho do título e dos tópicos estão adequados

2.10 As ilustrações estão expressivas e suficientes

2.11 O material (papel/impressão) está apropriado

2.12 O número de páginas está adequado

\begin{tabular}{cccccc}
\hline Escore por bloco & 68 & 62 & 32 & 5 & 130 \\
\hline Percentual por bloco & 40,48 & 37,00 & \multirow{2}{*}{$\begin{array}{c}7,00 \\
\text { Penc }\end{array}$} & $\begin{array}{c}2,9 \\
8\end{array}$ & 77,48
\end{tabular}

\begin{tabular}{ccc}
\hline IVC & 0,77 \\
\hline Total dos percentuais & 99,99 & 77,48 \\
\hline
\end{tabular}

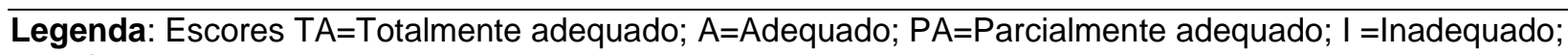
IVC=Índice de Validação de conteúdo.

Fonte: GALVAO RO, et al., 2020. 
Tabela 3 - Avaliação de Juízes Especialistas de Área Saúde na tecnologia educacional "Guia AVC para você e familiares", segundo relevância em Escala Likert.

\begin{tabular}{|c|c|c|c|c|c|}
\hline \multirow[t]{2}{*}{ Itens } & \multicolumn{3}{|c|}{$\begin{array}{c}\text { Escores }(\mathrm{N}=14) \text { : Escore } \\
\text { x100/ } \\
\text { TA+P+PA=Percentual } \\
\text { por escore }\end{array}$} & \multicolumn{2}{|r|}{$\begin{array}{l}\text { Índice de } \\
\text { concordân } \\
\text { cia por } \\
\text { item: TA+A } \\
\text { x } 100 \\
\end{array}$} \\
\hline & TA & A & PA & $\mathbf{I}$ & Total de Juízes \\
\hline \multicolumn{6}{|l|}{ Bloco 3. Relevâncias } \\
\hline $\begin{array}{l}3.1 \text { Os temas retratam aspectos- } \\
\text { chave que devem ser reforçados }\end{array}$ & 9 & 4 & 1 & 0 & 0,92 \\
\hline $\begin{array}{c}3.2 \text { O material permite a } \\
\text { transferência e generalização do } \\
\text { aprendizado a diferentes } \\
\text { contextos }\end{array}$ & 6 & 6 & 2 & 0 & 0,85 \\
\hline $\begin{array}{l}\text { 3.3 A TE propõe a construção de } \\
\text { conhecimentos }\end{array}$ & 5 & 6 & 3 & 0 & 0,78 \\
\hline $3.4 \mathrm{O}$ material aborda os & 6 & 5 & 3 & & \\
\hline $\begin{array}{c}\text { assuntos necessários para o } \\
\text { saber e o fazer do público-alvo } \\
\text { da TE }\end{array}$ & & & & 0 & 0,78 \\
\hline $\begin{array}{l}3.5 \text { Está adequado para ser } \\
\text { usado por qualquer profissional }\end{array}$ & 6 & 5 & 3 & 0 & 0,78 \\
\hline Escore por bloco & 32 & 26 & 12 & 0 & 58 \\
\hline Percentual por bloco & $45,7^{-}$ & 37,14 & 17,14 & 0 & 82,86 \\
\hline IVC & & & & & 0,83 \\
\hline Total dos percentuais & & 99 , & & & 82,66 \\
\hline
\end{tabular}

Legenda: Escores TA=Totalmente adequado; A=Adequado; PA=Parcialmente adequado; I =Inadequado; IVC=Índice de Validação de conteúdo.

Fonte: GALVAO RO, et al., 2020.

Tabela 4 - Avaliação de Juízes Especialistas de Área Não Saúde, na tecnologia educacional "Guia AVC para você e familiares" no Escore SAM.

\begin{tabular}{|c|c|c|c|c|c|c|c|c|c|c|c|c|c|c|}
\hline \multirow{2}{*}{$\begin{array}{l}\text { Bloco } \\
\text { Itens }\end{array}$} & \multicolumn{2}{|c|}{ Conteúdo } & \multicolumn{3}{|c|}{ Linguagem } & \multicolumn{3}{|c|}{ llustrações } & \multicolumn{3}{|c|}{ Motivacional } & \multicolumn{2}{|c|}{$\begin{array}{l}\text { Adequaçã } \\
\text { cultural }\end{array}$} & \multirow{2}{*}{$\begin{array}{l}\text { Escore } \\
\text { SAM }\end{array}$} \\
\hline & 1.1 & 1.2 & 1.3 & 2.1 & 2.2 & 2.3 & 3.1 & 3.2 & 4.1 & 4.2. & 4.3. & 5.1. & 5.2. & \\
\hline \multicolumn{15}{|l|}{ Juízes } \\
\hline $\begin{array}{l}\text { Direção de } \\
\text { arte }\end{array}$ & 2 & 2 & 2 & 2 & 2 & 1 & 2 & 2 & 2 & 2 & 2 & 2 & 2 & 25 \\
\hline Publicidade & 2 & 2 & 1 & 1 & 1 & 1 & 1 & 1 & 2 & 2 & 2 & 1 & 2 & 19 \\
\hline Design & 2 & 2 & 2 & 2 & 2 & 2 & 2 & 2 & 2 & 2 & 2 & 2 & 2 & 26 \\
\hline Pedagogia & 2 & 2 & 2 & 2 & 2 & 2 & 2 & 2 & 2 & 2 & 2 & 2 & 2 & 26 \\
\hline Design & 2 & 2 & 2 & 1 & 1 & 1 & 1 & 1 & 2 & 2 & 2 & 1 & 1 & 19 \\
\hline $\begin{array}{c}\text { Média } \\
\text { aritmética } \\
\text { simples }\end{array}$ & & & & & & & & & & & & & & 26 \\
\hline
\end{tabular}


Os juízes-especialistas ofereceram sugestões gerais: indicaram correções pontuais nas temáticas abordadas por conta da abrangência dos assuntos; concordaram que o guia era uma boa porta de entrada para a introdução de conteúdos de forma sucinta por ser para um público leigo; destacaram a importância do autocuidado do indivíduo acometido e a significância do trinômio pessoa-família/cuidador-profissionais de saúde, que traz resultados positivos a todos os indivíduos no quesito qualidade de vida pós-evento vascular.

As sugestões mais específicas foram: inclusão de tópicos pertinentes sobre alterações frequentes após acidente vascular encefálico; a profissional de fonoaudiologia aconselhou a inserção do tema disfagia por ser um transtorno comum; o profissional de fisioterapia sugeriu um tópico exclusivo sobre alteração de marcha, que apesar de já constar no tópico sobre alterações motoras, se ampliado, poderia contribuir mais; recomendaram revisão da língua portuguesa por um profissional, para melhorar a qualidade da comunicação com o público-alvo. No geral, consideraram positiva a iniciativa da abordagem multiprofissional em uma ação realizada durante um projeto de iniciação científica.

A segunda versão do guia ilustrado, que ficou pronta após a validação com os JE, reforçou as alterações advindas das afeções crônicas, e foi conduzida maior adequação linguística; foram trocadas todas as imagens para imagens de domínio público e ao final ficou com 25 páginas.

\section{DISCUSSÃO}

O AVC é uma das principais causas de incapacidade a longo prazo, com origens vasculares múltiplas e curso clínico que muda ao decorrer do tempo (STEIGER N e CIFU AS, 2016). O acidente encefálico provoca alterações motoras, sensoriais e cognitivas de acordo com o território vascular acometido e a extensão da lesão (BRASIL, 2013). Desse modo, o custo social-econômico desse evento vascular é alto, estimado em aproximadamente US $\$ 50$ bilhões de dólares americanos anual nos Estados Unidos contabilizado em atendimentos, prescrições e reabilitação (MAJERSIK JJ e WOO D, 2020).

Com a revisão de literatura, foi possível a seleção de evidências sobre as alterações desencadeadas pelo AVC, para serem abordadas no guia ilustrado, e foram delineadas para que o guia pudesse trazer informações amplas ao público geral. O Ministério da Saúde do Brasil destaca as seguintes manifestações desencadeadas pelo infarto cerebral: o déficit sensório-motor com disfagia, paralisia facial, fraqueza muscular, déficits de sensibilidade, alterações visuais, limitação de atividades motoras e funcionais; limitação de atividades da vida diária como alimentação, banho, higiene, vestuário de tronco superior e inferior; comunicação com afasia, dispraxia oral e de fala, disartria; distúrbios do humor; labilidade emocional com depressão; déficit cognitivo (BRASIL, 2013).

Assim, selecionaram-se os temas a serem discutidos no guia ilustrado, com foco nas manifestações mais frequente e diretamente relacionadas a promoção da qualidade vida. Regularmente, as pessoas após acidente vascular cerebral adquirem alterações residuais, não só perda parcial ou total motoras, mas também sensitivas, interrupção completa de movimentos voluntários musculoarticulares, dores difusas, comunicação insatisfatória e distúrbios de memória (COSTA FA, et al., 2011). Além de déficits funcionais, como fraqueza, afasia, negligência, alterações cognitivas ou restrições de participação social (GITTLER M e DAVIS AM, 2018).

Na primeira versão do guia, já se reforçava a atuação das diversas especialidades da área da saúde em relação as necessidades de reabilitação a serem desenvolvidas com interdisciplinaridade por uma equipe multiprofissional para se trabalhar os conhecimentos de forma integrada, do mesmo modo que devem envolver o paciente e a família na abordagem dos tratamentos, promovendo a autonomia de decisão (SCHMIDT MH, et al., 2019; LINDSAY LR, et al., 2020). Essa abordagem ampla na TE foi marcada positivamente pelos juízes validadores. Também se destacava o processo de cuidado contínuo, pois quanto mais afecções crônicas oriundas de possíveis períodos de agudização das patologias adquiridas, mais intervenções associadas as mudanças de estilo de vida são necessárias (BRASIL, 2013). 
Em relação a validação da primeira versão, os juízes-especialistas ressaltaram como positivo o tópico autocuidado. Cabe sobre isso ressaltar a importância do papel central pertencente ao indivíduo que vive o pós AVC, por conseguinte, as terapias devem ser voltadas para que tenha autonomia, na busca de reconstruir e proporcionar autorresponsabilidade na gestão da própria saúde e vida. Acentua-se também, o benefício desse envolvimento participativo com a família e o cuidador na integração com os profissionais de saúde (NUNES HJM, et al., 2017). Nesse caso, a tomada de decisão sobre qual será a abordagem de tratamento na reabilitação mais adequada não é correspondente ao profissional de saúde, mas o compete explanar as opções de tratamentos disponíveis naquela realidade para que o trinômio indivíduo-família/cuidadorprofissionais.

A segunda versão, elaborada após a validação, acatou as sugestões dos JE. Com a organização de um material educativo há que cuidar de aspectos de legibilidade com o intuito de favorecer a compreensão pelo público-alvo, por conseguinte, se acatou a sugestão quanto a revisão do texto (SIQUEIRA AF, et al., 2020). O guia foi expandido para contemplar também informações sugeridas sobre disfagia e alteração de marcha baseadas nas orientações do Guideline de AVC reabilitação e recuperação para adultos do Journal of the American Medical Association (JAMA) que traz como recomendação forte $1 \mathrm{~A}$ que pacientes devem ser treinados para vida diária de acordo com suas necessidades individuais no local onde receberam a alta, além de serem avaliadas precocemente tanto 1B para problemas de comunicação e disfagia, quanto $1 \mathrm{C}$ para equilíbrio (GLITTER M, et al., 2018).

A literatura indica que indivíduos após um AVC, podem manifestar disfagia como uma complicação comum na fase aguda, afeta entre $37 \%$ a $78 \%$, porém na fase crônico representa de $25 \%$ a $65 \%$ dos pacientes, dependendo do método de avaliação utilizado (WANG L, et al., 2019; SPROSON L, et al., 2018, ELTRINGHAM S, et al., 2018). Essa deficiência resulta de funcionamento anormal dos músculos da orofaríngea e esfíncter superior do esôfago, pode causar distúrbios de natureza oral, como déficit mastigatório, diminuições na manipulação do alimento e consequentemente na produção de bolus reduzida, e distúrbios de natureza faríngea como resíduos alimentares e respectivas aspirações. Em relação as complicações frequentemente associadas a disfagia são pneumonia aspirativa desnutrição, desidratação, hospitalização prolongada e mortalidade (ELTRINGHAM SA, et al., 2018). O tratamento da disfagia pós-AVC geralmente se concentra no manejo sintomático, ao qual o ocorre sim a modificação nas texturas dos alimentos para ingestão oral e a adaptação da postura corporal, mas não ocorre a reabilitação física direta da função da deglutição (SPROSON L, et al., 2018)

Algumas pessoas apresentam dependência da extremidade inferior não afetada pelo acidente vascular para permanecer em pé e deambular, demonstrando comprometimento do equilíbrio e consequentemente da marcha (CURUK E, et al., 2019). Os tipos de alterações mais comuns na marcha apresentam distinções no comprimento da passada, comprimento do passo, na velocidade da marcha, na fase de apoio curto e na fase de balanço relativamente longa do lado afetado, vindo a consumir de três a quatro vezes mais energia em relação a pessoas saudáveis (OH SJ, et al., 2019). A recuperação da capacidade da marcha sido considerada o principal objetivo da reabilitação em clientes vivendo com acidente encefálico (CARDA S, et al., 2013). Comumente, o tratamento dos distúrbios neurológicos de marcha envolve a caminhada em decline positivo ou negativo, treinamento de observação de ação (AOT) e observação de ação funcional (FAO) ou aparelho imobilizador de joelho combinado com a órtese imobilizadora de pé (PUNT, et al., 2017, CURUK E, et al., 2019, ALU B e BAZANCIR Z, 2017).

A neuroplasticidade das células de outras áreas do cérebro não afetadas pode assumir determinadas funções da área afetada, o que torna possível a reabilitação em alguns casos. Assim, há a forte recomendação de que a terapia seja continuada em casa ou outros locais, ao invés de serem interrompida no momento da alta, compatível com a proposta final de depois da validação o guia esteja disponível nas unidades de atenção secundárias e terciárias (SILVA IFG, et al., 2016). O acompanhamento a após alta pelos profissionais de saúde reforça o excelente suporte para a reabilitação e deve ser estimulada desde o momento da internação hospitalar como destacaram os juízes. (PAULA A, et al. 2019). Atividades de educação em saúde mediadas por tecnologias poderão potencializar as práticas dos profissionais de saúde junto a pessoas que, dentre outras condições, tiveram um AVC e requerem cuidados quaternários. 
A TE disponibiliza informações baseadas em evidencias para aplicação na vida diária, com vistas a qualidade de vida. No "Guia AVC para você e familiares", se sintetizam definições de doença, causas, sintomas mais comuns e tratamentos voltados a reabitação na fase crônica do acidente vascular encefálico.

\section{CONCLUSÃO}

O guia ilustrado revelou-se, posteriormente a validação, um instrumento mediador adequado tanto para ações, quanto para educação em saúde com pessoas no pós-AVC. A relevância desse estudo está relacionada à possibilidade de tornar as informações científicas mais acessíveis ao público-alvo e viabilizar técnicas seguras de reabilitação com foco multiprofissional, de modo a incentivar o autocuidado de pessoas em reconstrução de sua autonomia.

\section{AGRADECIMENTOS E FINANCIAMENTO}

À Universidade do Estado do Amazonas e Fundação de Amparo à Pesquisa do Estado do Amazonas pelo financiamento da pesquisa (Processo no 14961). Aos juízes pela disposição em participar da iniciativa, e também à Maria Oliene de Oliveira Galvão e Débora Oliveira Marques pelo incentivo.

\section{REFERÊNCIAS}

1. ALVES N, PAZ FAN. Análise das principais sequelas observadas em pacientes vítimas de acidente vascular cerebral - AVC. Revista da FAESF, 2018; 2(4):25-30.

2. BRASIL. 2017. In. Acidente vascular cerebral (AVC): Uma das principais causas de mortes no mundo, doença pode ser prevenida com hábitos saudáveis de vida, 2012. Brasília, Ministério da Saúde. 2017. Disponível em: http://www.brasil.gov.br/noticias/saude/2012/04/acidente-vascular-

cerebralavc?TSPD_101_R0=a07bffd046a97be2198de8f14fc828dbs5u00000000000000001c25d1b1ffff0000000000 $0000000000000000005 c a 1 c 342009 f b 71$ ed608282a9212ab200013e3ee87d40c8956abfa3c01ff960a0a8517ff81.

Acesso em: 1 mar. 2019.

3. BRASIL. Ministério da Saúde. Secretaria de Atenção à Saúde. Departamento de Ações Programáticas Estratégicas. Diretrizes de atenção à reabilitação da pessoa com acidente vascular cerebral. Brasília: Ministério da Saúde, 2013.

4. BRASIL. Ministério da Saúde. Secretaria de Vigilância em Saúde. Departamento de Análise de Situação de Saúde. Plano de Ações Estratégicas para o Enfrentamento das Doenças Crônicas Não Transmissíveis (DCNT) no Brasil 2011-2022. Brasília: Ministério da Saúde, 2011.

5. BRORSON JR, CIFU AS. Management of Patients With Acute Ischemic Stroke. JAMA, 2019; 322(8):777-778.

6. CARDA S, et al. Does altering inclination alter effectiveness of treadmill training for gait impairment after stroke? A randomized controlled trial. Clin Rehabil., 2013; 27(10):932-938.

7. COSTA FA, et al. Estado neurológico e cognição de pacientes pós-acidente vascular cerebral. Rev. esc. enferm. USP [online], 2011; 45(5):1083-1088.

8. CURUK E, et al. The Effect of Motor and Cognitive Tasks on Gait in People with Stroke. J Stroke Cerebrovasc Dis, 2019; 28(11):e104330.

9. ELTRINGHAM SA, et al. Impact of Dysphagia Assessment and Management on Risk of Stroke-Associated Pneumonia: A Systematic Review. Cerebrovasc Dis., 2018; 46(3-4):99-107.

10. GITTLER M, DAVIS AM. Guidelines for Adult Stroke Rehabilitation and Recovery. JAMA, 2018;319(8):820-82.

11. LINDSAY LR, et al. Updated Approach to Stroke Rehabilitation. Med Clin North Am., 2020; 104(2):e199-211.

12. MAJERSIK, et al. The enormous financial impact of stroke disability. Neurology, 2020; 94:377-378.

13. MALTA DC, et al. A implantação do Sistema de Vigilância de Doenças Crônicas Não Transmissíveis no Brasil, 2003 a 2015: alcances e desafios. Rev. bras. epidemiol.,2017; 20(4):661-675.

14. STEIGER N, CIFU AS. Primary Prevention of Stroke. JAMA, 2016; 316(6):658-659.

15. MOREIRA APA, et al. Jogo educativo de administração de medicamentos: um estudo de validação. Rev Bras Enferm, $2014 ; 67(4): 528-534$.

16. NASCIMENTO MHM, Teixeira E. Educational technology to mediate care of the "kangaroo family" in the neonatal unit. Rev Bras Enferm [Internet], 2018; 71(3):1290-1297.

17. NUNES HJM, QUEIROS PJP. Doente com acidente vascular cerebral: planeamento de alta, funcionalidade e qualidade de vida. Rev. Bras. Enferm., 2017; 70(2):415-423. 
18. $\mathrm{OH}$ SJ, et al. The effects of functional action-observation training on gait function in patients with post-stroke hemiparesis: A randomized controlled trial. Technol Health Care, 2019; 27(2):159-165.

19. OPAS/OMS Organização Pan Americana da Saúde em Brasília/Organização Mundial da Saúde. 2018. 10 principais causas de morte no mundo. Brasília: Organização Pan Americana da Saúde em Brasília. Disponível em: https://www.paho.org/bra/index.php?option=com_content\&view=article\&id=5638:10-principais-causas-de-morte-nomundo\&ltemid=0. Acesso em: 1 mar. 2019.

20. PAULA A, et al. Reabilitação pós-AVC: identificação de sinais e sintomas fonoaudiológicos por enfermeiros e médicos da Atenção Primária à Saúde. CoDAS, 2019;31(2):e20180015

21. PUNT M, et al. Do clinical assessments, steady-state or daily-life gait characteristics predict falls in ambulatory chronic stroke survivors? J Rehabil Med, 2017; 49(5):402-409.

22. SÁ BP, et al. Perfil de pacientes internados por Acidente Vascular Cerebral em hospital do Vale do Taquari/RS. Revista Neurociências, 2014; 22(3):381-387.

23. SCHMIDT MH, et al. Acidente vascular cerebral e diferentes limitações: uma análise interdisciplinar. Arq. Cienc. Saúde UNIPAR, 2019; 23(2):139-144.

24. SILVA IFG da, et al. Viver e Cuidar Após o Acidente Vascular Cerebral. Rev. Enf. Ref., 2016; ser IV(8): $103-111$.

25. SIQUEIRA AF, et al. Validation of a handbook on suicide prevention among students: talking is the best solution. Rev Rene. 2020; $21: e 42241$.

26. SPROSON L, et al. Combined electrical stimulation and exercise for swallow rehabilitation post-stroke: a pilot randomized control trial. Int J Lang Commun Disord.,2018;53(2):405-417.

27. TALU B, Bazancir Z. The effect of different ankle and knee supports on balance in early ambulation of post-stroke hemiplegic patients. Neurol Sci., 2017; 38(10):1811-1816.

28. TEIXEIRA E; OLIVEIRA, D.C. Representações sociais de educação em saúde em tempos de Aids. Rev Bras Enferm., $2014 ; 67(5): 810-817$.

29. TEIXEIRA E, et al. Arqueologia da educação em saúde: concepção e desenvolvimento de um campo disciplinar. Rehrevista educação e humanidades, 2020; I(1):291-305.

30. TEIXEIRA E, Nascimento MHM. Pesquisa Metodológica: perspectivas operacionais e densidades participativas. IN: Teixeira, E. (Org.). Desenvolvimento de tecnologias cuidativo-educacionais. Volume II. Porto Alegre: Moriá; 2019.

31. WANG Z, et al. Effects of capsaicin on swallowing function in stroke patients with dysphagia: A randomized controlled trial. J Stroke Cerebrovasc Dis., 2019; 28(6):1744-1751. 